\section{Superar el racismo oculto e interculturizar las universidades. Experiencias, avances y desafíos ${ }^{1}$}

Daniel Mato

Universidad Nacional de Tres

de Febrero. Consejo Nacional

de Investigaciones Científicas

y Técnicas. Argentina

dmato@untref.edu.ar
A 100 años de la Reforma Universitaria de 1918 /

Desafíos de gestión

RECEPCIÓN: 24/06/17

ACEPTACIÓN FINAL: 23/10/17

\section{Resumen}

Inevitablemente, los protagonistas de la Reforma Universitaria de 1918 percibieron el reto de "romper la última cadena que (...) nos ataba a la antigua dominación monárquica y monástica" desde las ideas de su época. Lejos estaban aún de que las luchas contra el racismo y otros legados coloniales alcanzaran la importancia que hoy poseen en las agendas de algunos movimientos sociales y sectores universitarios, incluso cuando todavía falta mucho por lograr. Este artículo analiza la oportunidad histórica y la necesidad ética, social, jurídica y epistemológica de que actualicemos y logremos ese reto planteado por la Reforma de 1918, que hoy podemos expresar en términos de superar todas las formas de racismo oculto (cultural, social, económico, ambiental, epistemológico) e interculturalizar las universidades. En América Latina existe actualmente más de un centenar de valiosas experiencias que muestran que estos avances son posibles. También muestran que estas transformaciones llevan a cuestionar los límites entre disciplinas, así como entre extensión, aprendizaje/docencia e investigación. Este artículo ofrece un panorama de las experiencias que algunas universidades "convencionales" han venido realizando junto con, y/o para, pueblos indígenas y afrodescendientes, así como las de otras universidades caracterizadas como "interculturales" y algunas creadas por organizaciones de estos pueblos. Adicionalmente, examina sus logros, avances y desafíos.
Palabras clave

- Universidad

- Racismo oculto

- Interculturalidad

- Pueblos indígenas

- Afrodescendientes

\section{Resumo}

Inevitavelmente, os protagonistas da Reforma Universitária de 1918 perceberam o desafio de "quebrar a última corrente que (...) nos amarrava à antiga dominação monárquica e monástica" desde os ideais de sua época. Ainda estavam longe de que as lutas contra o racismo e outros legados coloniais atingiram a importância que hoje possuem nas agendas de alguns movimentos sociais e setores universitários, mesmo quando ainda falta muito por conseguir.

Este artigo analisa a oportunidade histórica e a necessidade ética, social, jurídica e epistemológica de atualizarmos e conseguirmos esse desafio exposto pela Reforma de 1918, que hoje podemos expressar em termos de superar todas as formas de racismo oculto (cultural, social, econômico, ambiental, epistemológico) e interculturalizar as universidades. Existe atualmente na América Latina mais de uma centena de experiências valiosas que demonstram que estes avanços são possíveis. Evidenciam também que estas transformações levam a questionar os limites entre disciplinas, assim como entre extensão, aprendizagem/docência e pesquisa. Este artigo oferece um panorama das experiências que algumas universidades "convencionais" vêm realizando junto com, e/ou para, povos indígenas e afrodescendentes, assim como as de outras universidades caracterizadas como "interculturais" e algumas criadas por organizações destes povos. Além do mais, examina suas conquistas, avanços e desafios.

Palavras-chave

- Universidade

- Racismo oculto

- Interculturalidade

- Povos indígenas

- Afrodescendentes

\section{Para citación de este artículo}

Mato, D. (2017). Superar el racismo oculto e

interculturizar las universidades. Experiencias,

avances y desafíos.. Revista $+E$ versión en

línea, 7(7), 188-203. Santa Fe, Argentina:

Ediciones UNL. 


\section{Introducción}

Inevitablemente, los protagonistas de la Reforma Universitaria de 1918 percibieron el reto de "romper la última cadena que, en pleno siglo XX, nos ataba a la antigua dominación monárquica y monástica" desde las ideas de su época. No podría haber sido de otra manera. Pero faltaba mucho tiempo para que las luchas contra el racismo y otros legados coloniales y a favor de la valoración por las diferencias culturales alcanzaran el lugar que hoy ocupan en instrumentos jurídicos internacionales ${ }^{2}$ y en otros vigentes en la mayoría de los países latinoamericanos, así como en las agendas de algunos movimientos sociales y sectores universitarios. Pese a que debe destacarse que en lo que hace a las formas de racismo oculto todavía hay mucho por lograr. Este artículo analiza la oportunidad histórica y la necesidad ética, social, jurídica, y epistemológica de que actualicemos y superemos ese reto planteado por el movimiento de la Reforma Universitaria, el de romper con la dominación monárquica, que hoy podemos expresar en términos de superar todas las formas de racismo oculto e interculturalizar las universidades, para de este modo contribuir a superar el racismo de nuestras sociedades.

Aún hoy, la mayoría de las universidades latinoamericanas, como las políticas y los sistemas de evaluación y acreditación del sector, responde a anticuados formatos monoculturales - que son consecuencia del legado colonial eurocéntrico-y opera como reproductora de diversas formas de racismo oculto (cultural, social, económico, ambiental, epistemológico). Estos formatos institucionales monoculturales no son pertinentes respecto de la diversidad cultural propia de las sociedades de las que estas universidades forman parte y conducen a desconocer las visiones de mundo, proyectos de futuro, lenguas, valores, conocimientos, modos de aprendizaje y de producción conocimientos de pueblos indígenas, afrodescendientes y otros grupos sociales culturalmente diferenciados. Como se verá en las próximas páginas, esta limitación ya ha sido reconocida en la Declaración Final de la Conferencia Regional de Educación Superior realizada en Cartagena de Indias en 2008. Pero además, y relevante para esta publicación, ese modelo universitario, entre otros problemas, exhibe y fortalece anticuadas y entorpecedoras "barreras" entre extensión, aprendizaje/docencia e investigación. En contraste con ese modelo monocultural, en América Latina existe actualmente más de un centenar de valiosas experiencias que muestran que es posible avanzar en la superación de esas formas de racismo oculto, así como —algo muy importante para esta publicación— que esto conduce a cuestionar las mencionadas barreras entre extensión, aprendizaje/docencia, e investigación.

Este texto ofrece un panorama sintético de las experiencias de colaboración intercultural que algunas universidades "convencionales" 3 latinoamericanas han venido realizando junto con referentes, comunidades y organizaciones de pueblos indígenas y afrodescendientes, así como las de universidades creadas por organizaciones de estos pueblos y otras caracterizadas como "interculturales". Adicionalmente, se comentan algunos de sus principales logros y desafíos.

No obstante, antes de avanzar en la exposición de esos asuntos, pienso que resulta provechoso explicitar que mis investigaciones sobre estos tipos de experiencias de colaboración intercultural y las labores de acompañamiento a algunas de ellas que he venido realizando desde hace 15 años en varios países latinoamericanos me han llevado a concluir que quienes venimos del ámbito de las universidades "convencionales" tenemos mucho para aprender de ellas. Podemos aprender tanto de algunas innovaciones que vienen desarrollándose en el ámbito de las experiencias de colaboración entre universidades convencionales y pueblos indígenas y afrodescendientes como de los modelos universitarios que han creado algunas universidades indígenas e interculturales. Los aprendizajes en cuestión remiten a varias dimensiones de la vida universitaria pero, a apropósito de la temática de esta revista, parece importante destacar que tanto esas innovaciones al interior de universidades convencionales como esos otros modelos universitarios transgreden las fronteras entre docencia, investigación y extensión que caracterizan la vida de la mayoría de las universidades convencionales. Esto incluso les ha llevado a poner en cuestión las ideas unidireccionales de "extensión", en lo que significativamente convergen con algunas corrientes críticas de extensión universitaria que se han venido dando crecientemente en América Latina, especialmente desde la década de 1960. De hecho, en mi caso, el conocimiento cercano de esas experiencias de colaboración intercultural y de esos otros modelos de universidad me ha llevado a plantearme una línea de
1) Este artículo es una versión significativamente ampliada del artículo: Mato, D. (2016). Actualizar las propuestas de la Reforma Universitaria de 1918. Interculturizar la Educación Superior: experiencias, avances y desafíos, publicado en la revista Integración y Conocimiento (Núcleo de Es- tudios e Investigaciones en Educación Superior del MERCOSUR, Universidad Nacional de Córdoba), (5), 35-52. 2) Significativamente, la Declaración Universal de Derechos Humanos fue adoptada por la Organización de las Naciones Unidas en 1948, y la Convención Internacional sobre la
Eliminación de todas las Formas de Discriminación Racial en 1965. 3) En este artículo, como en publicaciones anteriores, denomino "convencionales" a las universidades que no han sido creadas por referentes $y / u$ organizaciones de pueblos indígenas o afrodescendientes, o para responder a sus demandas y propuestas. Desde luego, se trata solo de una denominación genérica a los fines de la exposición, por cuanto dentro del universo de las así llamadas "convencionales" cabe observar la existencia de diversos tipos de modelos universitarios. 
investigación específicamente dedicada a documentar y analizar cómo las experiencias de vinculación de equipos de universidades convencionales argentinas con diversos tipos de actores sociales enriquecen las oportunidades de docencia/aprendizaje e investigación (Mato, 2013a; 2013b y 2015).

\section{Racismo e inequidades asociadas en las sociedades latinoamericanas}

Las sociedades latinoamericanas contemporáneas están muy lejos de constituir ejemplos aceptables de democracia y equidad. Esto es especialmente inequívoco en lo que hace a la situación de los pueblos y personas indígenas y afrodescendientes que forman parte de estas sociedades (Banco Mundial 2015, CIDH-OEA 2011, IIPE-UNESCO, 2012). Las importantes inequidades que afectan particularmente las vidas de esos pueblos y sus miembros no solo son ética y jurídicamente cuestionables, sino que además comprometen las posibilidades de mejoramiento de la calidad de vida de cada una de las sociedades de las que forman parte. Las formas de sentido común predominantes entre la mayor parte de los miembros de estas sociedades ocultan este tipo de problemas. Ellas disimulan la persistencia de sensibilidades, creencias, sentimientos, ideas, políticas y prácticas racistas históricamente constitutivas de estas sociedades que, por lo general, perviven de manera solapada o inconsciente.

Las universidades y otras instituciones de educación superior (IES) deberían contribuir a resolver esas inequidades y problemas, pero en la mayoría de los casos están muy lejos de hacerlo. Esto se debe - en buena medida - a que son instituciones rígidamente monoculturales, cuyos programas de formación no incluyen los conocimientos, lenguas, visiones de mundo, propuestas de futuro, y modos de aprendizaje y de producción de conocimientos de esos pueblos. De manera semejante, en la mayoría de los casos la participación plena de miembros de esos pueblos en calidad de autoridades, funcionarios, docentes, o estudiantes resulta entre muy escasa y nula. ¿Qué se puede esperar de universidades con estas características? ¿Qué hacer para resolver estas deficiencias? La II Conferencia Regional de Educación Superior (CRES), realizada en Cartagena de Indias en 2008, reconoció la importancia de estas deficiencias y problemas, al punto que en su Declaración Final incluyó dos acápites específicos al respecto que conviene citar in extenso:
"C-3: Se deben promover la diversidad cultural y la interculturalidad en condiciones equitativas y mutuamente respetuosas. El reto no es sólo incluir a indígenas, afrodescendientes y otras personas culturalmente diferenciadas en las instituciones tal cual existen en la actualidad, sino transformar a éstas para que sean más pertinentes con la diversidad cultural. Es necesario incorporar el diálogo de saberes y el reconocimiento de la diversidad de valores y modos de aprendizaje como elementos centrales de las políticas, planes y programas del sector".

"D-4: La Educación Superior, en todos los ámbitos de su quehacer, debe reafirmar y fortalecer el carácter pluricultural, multiétnico y multilingüe de nuestros países y de nuestra región". (2008, http://www.unesco.org.ve/index.php?option=com content\&view=article\&id=365\&ltemid=423\&lang=es)

La importancia de responder apropiadamente a estos desafíos fue explicitada reiterada en la "Declaración de Panamá sobre la Educación en la Sociedad del Conocimiento" ", emitida en junio de 2012 como resultado de la "Conferencia Interparlamentaria sobre La Educación en la Sociedad del Conocimiento". Esta Declaración, suscrita por los Presidentes de las Comisiones de Educación o equivalentes de los Parlamentos miembros del Parlatino, expresa textualmente que los firmantes de la misma

"suscriben e impulsan la Iniciativa Latinoamericana por la Diversidad Cultural y la Interculturalidad con Equidad en Educación Superior, emanada del 'Taller Regional de Políticas de Educación Superior y Pueblos Indígenas y Afrodescendientes en América Latina y el Caribe', realizado en la Universidad de Panamá los días 24 y 25 de mayo del 2012, convocado por UNESCO/IESALC" .

Estos reconocimientos de la importancia los desafíos que debe encarar la Educación Superior en América Latina son resultado del desarrollo de varios procesos sociales significativos. Uno de ellos suele nombrarse como el de reconocimiento de la "deuda histórica" que estas sociedades tienen con los pueblos indígenas y afrodescendientes. Este reconocimiento ha ido avanzado gracias a las luchas de estos pueblos, las cuales históricamente contaron con el apoyo de algunos movimientos sociales internacionales, como los organizados para luchar contra con el racismo y por los derechos humanos, y más recientemente también por los
4) Ver: http://virtualeduca.org/documentos/2012/declaracion-panama-2012.pdf
5) Ver: http://www.iesalc.unesco.org. ve/index.php?option=com_content\&view=article\&id=2973: presentan-ini- ciativa-latinoamericana-por-la-diversidad-cultural-y-la-interculturalidad-con-equidad-en-educacion-su- perior\&catid=100:en-portada\&ltemi$\mathrm{d}=449$ \&lang $=e n$ 


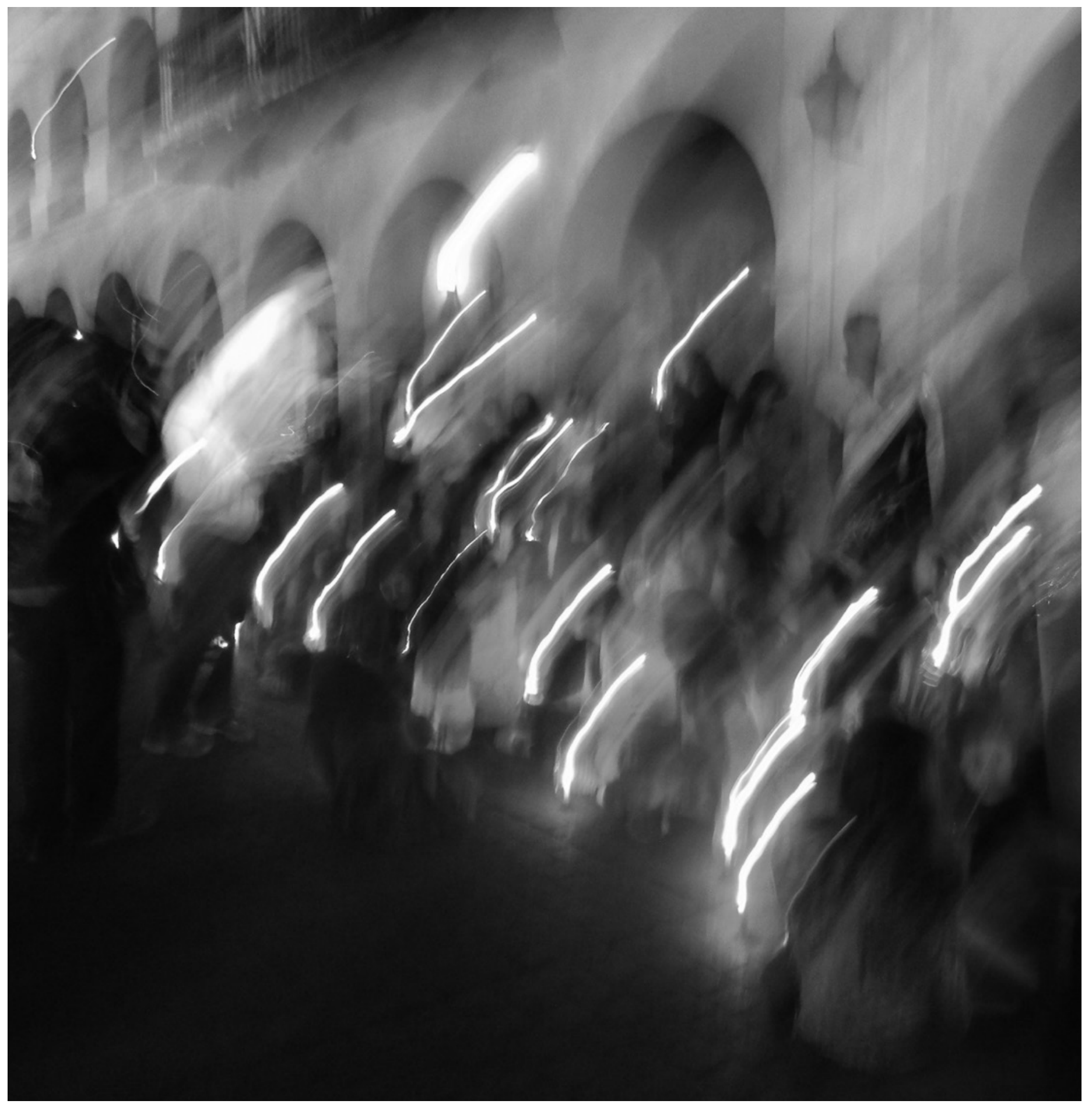

() Cecilia lucci 
movimientos sociales ambientalistas. Como resultado de estas luchas, se fue avanzado en la sanción de varias declaraciones e instrumentos jurídicos internacionales importantes, los cuales a su vez contribuyeron a lograr algunas reformas constitucionales que establecieron derechos explícitos para estos pueblos en numerosos países. ${ }^{6}$

Otro proceso que ha resultado significativo para lograr los reconocimientos expresados en la Declaración Final de la CRES 2008 y en la Declaración de Panamá ha sido el de la creciente la visibilidad cuantitativa de la importancia demográfica de estos pueblos, la cual ha venido haciéndose evidente desde que los censos nacionales incluyen información al respecto. Así, con base en las últimas rondas censales, según información publicada por la División de Población (CELADE) de la Comisión Económica para América Latina y el Caribe y por el Banco Mundial, la población indígena en América Latina comprende un total de 42 millones de personas y la afrodescendiente de 123 millones de personas. Es decir que ambas combinadas alcanzan a 165 millones de personas, y representan más del $28 \%$ de la población total de la región. ${ }^{7}$ Estos valores seguramente serían aún mayores de no mediar problemas de subregistración que han sido reiteradamente señalados por las organizaciones de estos pueblos. En cualquier caso, resulta interesante destacar que si bien la distribución de estas poblaciones varía entre países, los porcentajes de estos grupos de población respecto de los respectivos totales nacionales resultan significativos incluso en países en los que su importancia demográfica suele ser negada. Por ejemplo, e incluso obviando los problemas de subregistración ya señalados, en Chile, la población indígena representa el $6 \%$ del total nacional, en Colombia y Venezuela el $3 \%$, en Argentina y Costa Rica al 2,5 \%, en Paraguay el 1,7\% y en Brasil el 0,5\%. Estos porcentajes resultan mayores en Bolivia y Guatemala donde supera el $40 \%$, Perú donde alcanza al $26 \%$, o en México donde representa el $15 \%$, Panamá el $12 \%$, Honduras y Ecuador el $7 \%$ y Nicaragua el $6 \% .{ }^{8}$ Por otra parte, con base en las mismas fuentes y con los mismos reparos de subregistración, cabe afirmar que en los casos de Brasil y Venezuela la población afrodescendiente supera el $50 \%$, en tanto en Cuba representa el $36 \%$, en Colombia el $10 \%$, en Panamá el $9 \%$, en Costa Rica y Uruguay el $8 \%$, en Ecuador el 7,2 \%, en Honduras el $1 \%$, en Nicaragua el 0,5\%, en Guatemala el 0,4 \%, en Argentina el 0,3\%, en Bolivia y Paraguay el 0,2 \% y en el Salvador $0,1 \% .^{9}$

Otro proceso que también ha favorecido el reconocimiento de los mencionados desafíos que enfrenta la educación superior está asociado al desarrollo creciente de movimientos intelectuales críticos del modelo civilizatorio usualmente denominado occidental moderno, los cuales han venido insistiendo en que los graves problemas ambientales y sociales que afectan a nuestro planeta y a nuestra especie no solo están asociados de manera genérica a dicho modelo civilizatorio, sino también específicamente al modelo científico que le es propio. Esto ha llevado a la creciente aceptación de que los retos civilizatorios planteados demandan abandonar el credo cientificista monocultural para avanzar hacia posiciones de pluralismo epistemológico.

Finalmente, los reconocimientos de la importancia los desafíos que debe encarar la Educación Superior en América Latina también han estado asociados al creciente desarrollo y visibilidad de más de un centenar de valiosas experiencias que han venido desarrollándose en la región desde hace más de dos décadas, de los diversos tipos que se analizan más adelante en este artículo.

Es necesario superar esos modelos monoculturales y transformar a las universidades y otras IES en consonancia con lo recomendado en los acápites C3 y D4 de la Declaración Final de la CRES 2008 antes citados, los que además responden a lo establecido en el Convenio N ${ }^{\circ} 169$ de la Organización Internacional del Trabajo, que ha sido suscrito por 14 países latinoamericanos, para los cuales tiene rango constitucional. Límites de extensión impiden citar in extenso los artículos que aplican al caso, por lo que citaré solo algunos pasajes de especial interés: El artículo 26 dictamina que:
6) El Convenio 169 de la Organización Internacional del Trabajo (OIT) es uno de los instrumentos internacionales más importantes respecto del tema que nos ocupa, y ya ha sido ratificado por catorce países latinoamericanos: Argentina, Bolivia, Brasil, Chile, Colombia, Costa Rica, Ecuador, Guatemala, Honduras, México, Nicaragua, Paraguay, Perú y Venezuela. Tal como lo dispone este Convenio y las constituciones nacionales de los países antes mencionados, los Estados que han ratificado este instrumento internacional están obligados a acatar sus regulaciones. No es posible aquí entrar en detalles, pero como mínimo cabe señalar que los artículos 22, 26 y 27 del Convenio 169 consagran derechos específicos a los pueblos indígenas en materia de "educación a todos los niveles". Otros instrumentos internacionales relevantes son la Convención Internacional para la Eliminación de todas las Formas de Discriminación Racial (1965), la Convención Internacional sobre Derechos Económicos, Sociales y Culturales (1966), la Declaración de las Naciones Unidas sobre los Derechos de las Personas Pertenecientes a Mi- norías Étnicas, Religiosas y Lingüísticas (1992), la Declaración Universal de la UNESCO sobre Diversidad Cultural (2001), la Convención de la UNESCO sobre la Protección y Promoción de la Diversidad de las Expresiones Culturales (2005), la Declaratoria de Naciones Unidas de la Segunda Década de los Pueblos Indígenas 2005-2015, y la Declaración Universal sobre los Derechos de los Pueblos Indígenas (2007).

7) Fuentes: Totales nacionales: CELADE. Revisión 2015. Datos de poblaciones afrodescendientes: http:// www.alapop.org/alap/Serie-E-Inves-
tigaciones/N4/FINAL_Alap2015_Serie_elnvestigaciones_14012016.pdf Datos de pueblos indígenas: Banco Mundial, Latinoamérica Indígena en el Siglo XXI, 2015).

8) Las mencionadas fuentes no ofrecen datos respecto de Cuba, Haití, Uruguay y República Dominicana. 9) Las mencionadas fuentes no ofrecen datos respecto de Chile, México, Perú, Haití y República Dominicana. 
"deberán adoptarse medidas para garantizar a los miembros de los pueblos interesados la posibilidad de adquirir una educación a todos los niveles, por lo menos en pie de igualdad con el resto de la comunidad nacional".

El artículo 27 dispone que:

"la autoridad competente deberá asegurar la formación de miembros de estos pueblos y su participación en la formulación y ejecución de programas de educación [y que] los gobiernos deberán reconocer el derecho de esos pueblos a crear sus propias instituciones y medios de educación, siempre que tales instituciones satisfagan las normas mínimas establecidas por la autoridad competente en consulta con esos pueblos".

\section{El artículo 31 establece:}

"Deberán adoptarse medidas de carácter educativo en todos los sectores de la comunidad nacional (...), con el objeto de eliminar los prejuicios que pudieran tener con respecto a esos pueblos". ${ }^{10}$

Varios países latinoamericanos ya han dado algunos pasos en la materia, otros, como Argentina, se encuentran sumamente atrasados, especialmente en lo que respecta a Educación Superior. En Argentina y otros países se ha avanzado en otros niveles educativos pero muy poco en el Superior. Esto estimula una brecha entre niveles que además de ser injusta alienta conflictos. Tomando el caso argentino como referencia, cabe observar que los artículos 52 y 53 de la Ley de Educación Nacional (Ley N²6.206) permiten prever que las universidades continuarán recibiendo egresados del sistema de Educación Intercultural Bilingüe. El artículo 52 establece:

"La Educación Intercultural Bilingüe es la modalidad del sistema educativo de los niveles de Educación Inicial, Primaria y Secundaria que garantiza el derecho constitucional de los pueblos indígenas, conforme al art. 75 inc. 17 de la Constitución Nacional, a recibir una educación que contribuya a preservar y fortalecer sus pautas culturales, su lengua, su cosmovisión e identidad étnica". ${ }^{11}$

No obstante, las universidades nada ofrecen a los egresados de ese sistema intercultural bilingüe. La situación es análoga en otros países, aunque en algunos existen importantes experiencias a tomar en cuenta para avanzar en la materia. Con este fin, en este artículo se ofrece un panorama de las experiencias que algunas universidades y otras IES "convencionales" latinoamericanas han venido realizando — según los casos_ junto con, o para, pueblos indígenas y afrodescendientes, así como las de otras universidades e IES caracterizadas como "interculturales", y otras creadas por organizaciones de estos pueblos.

Es un deber destacar que el panorama y análisis que ofrezco en las páginas siguientes se basa en parte en las investigaciones sobre el tema que de manera individual he venido realizando desde 2004 , pero muy especialmente en las realizadas entre 2007 y 2011 con la colaboración de 70 colegas de diez países latinoamericanos, desde el Proyecto Diversidad Cultural e Interculturalidad en Educación Superior, del Instituto Internacional de la UNESCO para la Educación Superior en América Latina y el Caribe (Mato, coord., 2008, 2009a, 2009b, 2012),y en las realizadas desde 2011 desde el Programa Educación Superior y Pueblos Indígenas y Afrodescendientes en América Latina (Programa ESIAL) de la Universidad Nacional de Tres de Febrero, en colaboración con representantes de casi cincuenta universidades de nueve países latinoamericanos con quienes venimos trabajando en el marco de la Red Inter-universitaria Educación Superior y Pueblos Indígenas y Afrodescendientes en América Latina (Red ESIAL), creada con el apoyo de UNESCO-IESALC (Mato, 2015, 2016, 2017). ${ }^{12}$

\section{Valorar la diversidad cultural y promover relaciones interculturales equitativas}

Lo que en 1918 aparentemente no era siquiera pensable y menos aun susceptible de ser incluido en un programa de acción, un siglo después es inexcusablemente imperativo: las universidades deben reconocer y valorar la diversidad cultural y promover relaciones interculturales equitativas y mutuamente respetuosas, tanto en su seno como en las sociedades de las que forman parte. Este carácter imperativo deriva de los avances plasmados tanto en las constituciones nacionales de la mayoría de los países latinoamericanos, como en instrumentos jurídicos internacionales y en las declaraciones consensuadas entre representantes de universidades de toda la región.

La muy escasamente atendida meta de que las universidades y otras IES incluyan personas indígenas y afrodescendientes (como autoridades, funcionarios, docentes y estudiantes) dentro de su vieja institucionalidad no es suficiente. Es necesario ir más allá. Para superar ese pesado legado colonial que las caracteriza (con todas sus consecuencias, que no son solo de exclusión de personas, sino también de conocimientos, lenguas, formas de
10) http://www.ilo.org/wcmsp5/groups/ public/@ed_norm/@normes/documents/publication/wcms_100910.pdf 11) http://www.me.gov.ar/doc_pdf/ ley_de_educ_nac.pdf 12) Todos los libros resultantes de la labor realizada desde UNESCO-IESALC y desde UNTREF se encuentran disponibles tanto en versión impresa como digital, estas últimas en los sitios de dichas instituciones en Internet: http://www.iesalc.unesco.org.ve/ index.php?option=com_content\&view $=$ article\&id=22\&ltemid=405\&lan$\mathrm{g}=\mathrm{es}$ y http://untref.edu.ar/sitios/ciea/ biblioteca/ 
producción de conocimiento, modalidades de aprendizaje, como de modos de relación con las sociedades), las universidades deben reformarse a sí mismas para ser más pertinentes con la diversidad cultural propia de la historia y el presente de las sociedades de las que forman parte. Deben incluir las visiones de mundo, lenguas, conocimientos, modos de aprendizaje, modos de producción de conocimientos, sistemas de valores, y demandas de pueblos y comunidades indígenas y afrodescendientes, así como, según los países, de otros grupos culturalmente diferenciados.

Este ha sido, desde hace varias décadas, el planteo de numerosas expresiones y dirigentes de los movimientos indígenas y afrodescendientes de América Latina. También lo ha sido de diversos y numerosos sectores sociales e intelectuales que sin ser indígenas ni afrodescendientes comprendemos que no solo no es ético sostener modelos societarios y educativos que en la práctica excluyen a amplios sectores de población, sino que además entendemos que para las respectivas sociedades nacionales no es ni política, ni social, ni económicamente viable privarse de las importantes contribuciones de esas vertientes particulares, de su historia y de su presente. No se trata únicamente de "incluir" a personas de estos pueblos como individuos indiferenciados, sino de que las universidades y otras IES se transformen al incorporar apropiadamente sus lenguas, visiones de mundo, conocimientos, modos de aprendizaje y de producción de conocimientos, valores y proyectos societarios.

La valoración de la diversidad cultural y el desarrollo de relaciones interculturales equitativas y mutuamente respetuosas pueden ser recursos provechosos para mejorar la calidad de vida y el desarrollo humano sostenible de nuestras sociedades. Es curioso (y preocupante), pero algunas dirigencias universitarias parecen no acabar de valorar algo que ya ha sido comprendido y está siendo económicamente explotado por laboratorios farmacéuticos, agroindustrias y otras corporaciones transnacionales: los conocimientos tradicionales de esos pueblos. Al mencionar esto, de ningún modo sugiero que se trate de explotar esos conocimientos, sino que procuro llamar la atención respecto de que incluir esos conocimientos en los programas universitarios de ningún modo supone "hacerles un favor a unos pobrecitos excluidos". Se trata de reconocernos como ciudadanas/os de sociedades y Estados pluriculturales y plurilingües. Se trata de no vivir ignorando componentes y aspectos de nuestras propias sociedades para poder transformarlas de modos acordes con la diversidad que las caracteriza, y no como deformes reflejos de representaciones idealizadas de algunas sociedades europeas. En esto las universidades, y más en general la educación superior, tienen un papel importante que cumplir, no sólo como instituciones productoras de conocimiento, sino también como instituciones formadoras de cuadros técnicos y profesionales y de ciudadanos y dirigentes sociales, con capacidad de reflexión crítica. En contraste con ese deber ser y esa potencialidad, resulta preocupante que en pleno siglo XXI aún sean pocas las IES de la región cuya misión institucional y/o cuyo currículo incluyan la valoración de la diversidad cultural y la promoción de relaciones interculturales equitativas y mutuamente respetuosas. Esta situación resulta más preocupante si se piensa que la mayoría de las universidades y otras IES está a la zaga de instrumentos internacionales vigentes, como la Convención Internacional para la Eliminación de todas las Formas de Discriminación Racial (1965), la Convención Internacional sobre Derechos Económicos, Sociales y Culturales (1966), el Convenio No 169 de la Organización Internacional del Trabajo (1989), la Declaración de las Naciones Unidas sobre los Derechos de las Personas Pertenecientes a Minorías Étnicas, Religiosas y Lingüísticas (1992), la Declaración Universal de la UNESCO sobre Diversidad Cultural (2001), la Convención de la UNESCO sobre la Protección y Promoción de la Diversidad de las Expresiones Culturales (2005), La Declaración y el Programa de Acción de Durban (2009), la Declaratoria de Naciones Unidas de la Segunda Década de los Pueblos Indígenas 2005-2015, la Declaración Universal sobre los Derechos de los Pueblos Indígenas (ONU, 2007) y la Declaratoria y Plan de Acción 2015-2024 de Naciones Unidas del Decenio Internacional de los Afrodescendientes (2013). Nótese que el primero de estos instrumentos internacionales data del año 1965. Resulta muy preocupante que las universidades y otras IES tengan más de medio siglo de atraso respecto de instrumentos acordados por la mayoría de los gobiernos del planeta. No es un atraso menor, particularmente cuando se supone que deberían jugar papeles de avanzada.

No obstante, pese a ese rezago histórico, tal vez haya motivos para ser optimistas. La ya mencionada Conferencia Regional de Educación Superior (CRES-2008), en la cual participamos más de 3500 integrantes de la comunidad académica regional (incluyendo directivos, docentes, investigadores, estudiantes, funcionarios, representantes de organismos nacionales, regionales e internacionales y otros interesados en Educación Superior) emitió una Declaración Final que incluye los antes mencionados acápites C3 y D4 que contienen algunos valiosos planteamientos sobre el tema. Esto lleva a pensar que estas ideas comienzan a ser más ampliamente aceptadas y que hay más posibilidades de que sean puestas en práctica.

\section{No hay saber "universal", la colaboración intercultural es imprescindible}

Parece conveniente destacar la importancia y alcance de la idea central del acápite C3 de la Declaración Final de la CRES, 2008. Tal como explícitamente lo expresa, el reto no es simplemente "incluir a indígenas, afrodescendientes y otras personas culturalmente diferenciadas en las instituciones tal cual existen 
en la actualidad, sino transformar a éstas para que sean más pertinentes con la diversidad cultural". El reto es transformar a las universidades y a las políticas, planes y programas de educación superior y de ciencia y tecnología. Todavía hoy, se elaboran y aplican políticas de ciencia, se realiza investigación científica, y se imparte formación universitaria, en el marco de ciertas creencias (sí, creencias, pues no se trata de demostraciones "objetivas") según las cuales existirían dos clases de saber, uno solo de los cuales tendría validez universal: el científico, mientras que "el otro" (así homogeneizado, pese a que es notablemente diverso) únicamente tendría valor local. Es posible que esto sea "verdad" para las leyes físicas, pero de ningún modo lo es para campos de conocimiento como las ciencias sociales y las humanidades; como tampoco en lo que hace campos interdisciplinarios como los de salud y medio ambiente, entre otros. Existe cuantiosa bibliografía producida desde el propio sistema científico-académico que muestra que hay mucho por aprender al respecto de esos otros tipos de conocimientos y modos de producción de conocimiento. En pleno siglo XXI no es posible continuar ignorando que la idea de que "la ciencia" constituiría un saber de validez "universal" está directamente asociada al proceso histórico que se inició con la expansión militar y comercial de algunos Estados europeos sobre el resto del planeta, expandiendo sus propias creencias, visiones de mundo, e instituciones jurídicas, económicas y políticas. Esta expansión europea dio lugar al establecimiento de relaciones coloniales y se sostuvo largo tiempo asegurada por ellas. El problema que aun hoy estamos viviendo es que la ruptura de las relaciones coloniales y la fundación de las repúblicas no acabaron por completo con las formas de subordinación de los pueblos indígenas de América y de los numerosos contingentes de población africana traída a América en condiciones de esclavitud, como tampoco con las que aún hoy afectan a sus descendientes, incluyendo a grupos de población "mestizos" cuyos modos de vida y oportunidades económicas han sido especialmente marcados por esas relaciones históricas. Las relaciones jerárquicas entre dos tipos de saber, uno pretendidamente universal y otro local, son parte de estas dinámicas.
La descalificación de los modos de producción de conocimiento y acumulaciones de conocimientos de los pueblos indígenas y de los descendientes de las poblaciones africanas esclavizadas es parte de esa herencia colonial y racista, la cual debe ser estudiada más a fondo, criticada y superada. La necesidad de profundizar en el estudio de las consecuencias de esta herencia y de superarla no debe entenderse como una tarea limitada a quienes escogen formarse en antropología e historia, pues la ignorancia al respecto afecta la calidad de la formación de profesionales de muchos otros campos, como, por ejemplo, los de salud, derecho, geografía, agronomía, economía, ciencias políticas, minería, y arquitectura, entre otros. La colaboración intercultural en las universidades no puede limitarse a las escuelas de antropología. Debe partir del reconocimiento del valor de los conocimientos de los pueblos indígenas y poblaciones afrodescendientes para todos y cada uno de los campos de formación profesional. Más aún, no deberían estudiarse esas instituciones de manera aislada, sino cada una como parte de las respectivas visiones de mundo y modos de organización social (Macas 2001, Universidad Intercultural Amawtay Wasi 2004).

La hegemonía, y con ella la transferencia no reflexionada de ciertas creencias y valores de las llamadas "ciencias duras" a las llamadas "ciencias sociales" conduce a ignorar cómo nuestra subjetividad es constitutiva de nuestro trabajo de investigación. Por ejemplo, condiciona el planteamiento del problema/asunto a estudiar, la formulación de las preguntas de investigación, el establecimiento de la perspectiva de análisis, así como las relaciones con los actores "sobre", o "con", quienes estudiamos. Todo esto depende de desde dónde investigo, para qué investigo y qué pienso hacer con los resultados de la investigación. Pero estas preguntas no siempre se plantean. En muchos casos, los temas y perspectivas vienen dados de manera "natural" por las visiones que encarnan las revistas académicas en las que se aspira a publicar, o las instituciones que otorgan fondos para investigación. De este modo los resultados resultan marcados a priori por una suerte de ilusión objetivista ingenua, según la cual para asegurar tal objetividad se hace recomendable mantener cierta "distancia" respecto de 
los procesos sociales estudiados. Este factor de "distancia" es origen de una significativa diferencia entre el saber considerado "científico" y el que producen, por ejemplo, aquellos intelectuales indígenas que mantienen relaciones con sus comunidades, o bien los que producen los equipos de universidades "convencionales" que trabajan en colaboración con comunidades de pueblos indígenas y afrodescendientes, o los de las universidades "propias" de estos pueblos. Estas relaciones alimentan las preguntas y perspectivas a partir de las cuales producen sus conocimientos. Algo semejante ocurre con los equipos de cualquier universidad "convencional" que trabajan en proyectos que articulan extensión, docencia e investigación, como ha sido reportado por numerosos equipos universitarios (ver, por ejemplo, Almeida et al., 2011; FAUBA, 2015) y he documentado en publicaciones resultantes de mis investigaciones al respecto (Mato, 2013a y 2013b).

Unos y otros modos de producción de conocimientos, todos los conocimientos, el que se produce a partir de métodos "distanciados", como el que se produce a partir de relaciones estrechas de colaboración con otros actores, están marcados por las condiciones en que son producidos. Por eso la valoración y evaluación de los resultados de cualquier forma de producción de conocimiento debe hacerse tomando en cuenta esas condiciones de producción. No hay saber "universal", ninguno lo es, todos son relativos a las condiciones en que son producidos, por eso esas condiciones de producción deben ser claramente explicitadas; de manera análoga a cómo se hace al comunicar los resultados de investigaciones experimentales, aquellas que —como su nombre lo indica- resultan de experimentos, de laboratorios. Por estas razones la colaboración entre diversas formas de conocimiento es imprescindible.

Las consecuencias del modelo universitario monocultural afectan no solo a las poblaciones de origen indígena y afrodescendiente de las sociedades latinoamericanas, sino a cada una de las sociedades nacionales en su totalidad, incluyendo tanto a las poblaciones de origen netamente europeo, como a las caracterizadas como "mestizas". La negación consciente o inconsciente de la condición pluricultural de todas las sociedades latinoamericanas constituye un significativo lastre histórico, por lo que implica en términos de nuestra ignorancia acerca de nosotros mismos. Esta carga afecta no sólo las posibilidades de construir sociedades más justas e incluyentes, sino también las de que cada una de estas sociedades pueda utilizar todos los saberes y talentos a su alcance para construir su presente y futuro. Este problema no se resuelve con la celebración folclorizante de la diversidad, ni con la "nacionalización" de ritos, danzas y costumbres de estas poblaciones, incorporándolos a las festividades, monumentos y rituales de Estado. Tampoco se resuelve celebrando la "diversidad cultural”, mientras continúan violándose los derechos de esos grupos de población, menoscabando su condición humana, desvalorizando sus visiones de mundo, lenguas, conocimientos y modos de conocimiento (así esto se haga sea pasivamente, como ocurre con las formas de racismo oculto), en lugar de honrar sus derechos a restituciones territoriales, o, más grave aún, permitiendo (o fomentando) el avance de las explotaciones petroleras y mineras en sus territorios ancestrales.

Las situaciones contemporáneas respecto de estos problemas varían mucho de una sociedad latinoamericana a otra, pero en todas ellas estos problemas y este conflicto entre modos de producción de conocimiento afectan a la producción, circulación, apropiación y aplicación de conocimientos. Es decir, afectan la calidad de las labores de investigación y formación que realizan las universidades y otros tipos de IES. Por ello, cualesquiera que sean las vías específicas de solución de estos conflictos, la construcción de formas duraderas y provechosas de colaboración intercultural entre actores sociales con diversos tipos de conocimientos, formas de producirlos y modalidades de aprendizaje, ha de ser parte imprescindible de los caminos a transitar.

Pero, si bien en algunos casos los diversos tipos de conocimientos pueden resultar complementarios, en otros podrían estar en conflicto. La colaboración intercultural en la producción de conocimiento no es una panacea, hay conflictos. Por esto mismo, conviene identificarlos, analizarlos, y coproducir formas de manejarlos. Sin colaboración intercultural en la producción de conocimientos sobre nosotros mismos, en tanto agregados sociales, es imposible comprender nuestra experiencia social. Sin esa colaboración intercultural la comprensión de significativos aspectos históricos, jurídicos, políticos, económicos, sociales, y otros, de nuestras sociedades, será siempre sesgada y parcial. Sin tal colaboración, tal comprensión partirá de un "como si". Por esto, la colaboración intercultural en la producción de conocimientos sobre nuestras sociedades es imprescindible. Afortunadamente, este tipo de colaboración tiene numerosos y significativos antecedentes, como los que muestran cerca de un centenar de experiencias que han sido documentadas desde las dos iniciativas de investigación y colaboración interinstitucional de amplio alcance en las cuales se basa este artículo.

\section{Educación superior, pueblos indígenas y afrodescendientes en América Latina: panorama sintético}

Una característica saliente de este campo es la existencia de una amplia diversidad de experiencias. Esto no resulta sorprendente si se tiene en cuenta que las mismas han sido impulsadas por referentes y organizaciones de pueblos indígenas y afrodescendientes muy diversos entre sí. Más aún, estas tienen lugar en distintos países, en cada uno de los cuales la historia y presente de las relaciones entre dichos pueblos, los respectivos Estados y otros sectores sociales exhiben sus propias particularidades. 


\section{6}

Las consecuencias del modelo universitario monocultural afectan a cada una de las sociedades nacionales en su totalidad. La negación consciente o inconsciente de la condición pluricultural de todas las sociedades latinoamericanas constituye un significativo lastre histórico

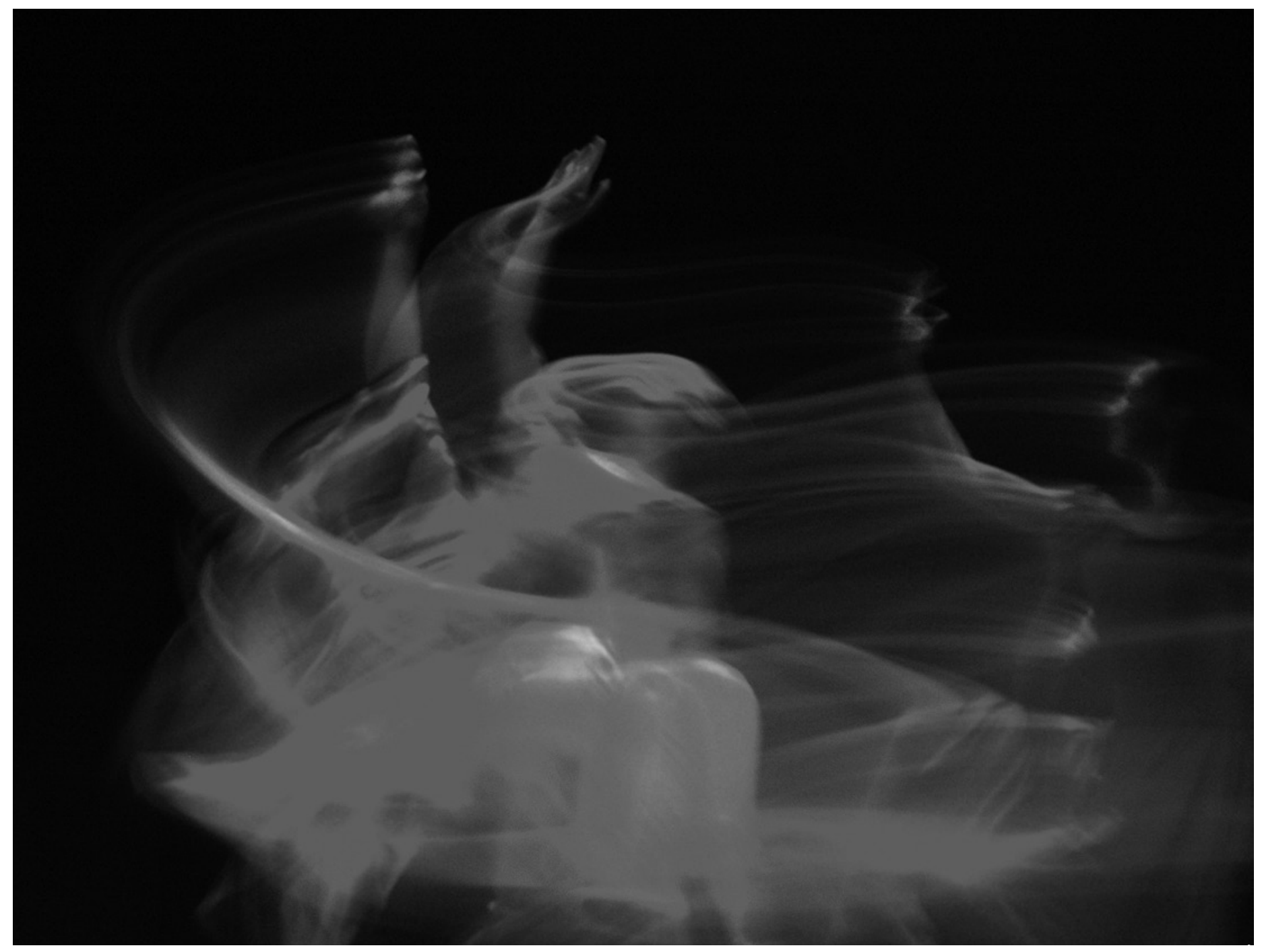

(c) Oscar Dechiara 
Dentro de este amplio y diverso campo de experiencias es posible distinguir entre cinco tipos principales de modalidades institucionales. A continuación, presento esquemáticamente esos diferentes tipos, por limitaciones de extensión no referiré ejemplos ilustrativos, sobre los cuales puede obtenerse información en las publicaciones resultantes de los dos proyectos de amplio alcance antes mencionados (Mato, 2008; 2009a; 2009b; 2015; 2016). En cambio, para cada tipo apunto algunos aspectos significativos respecto del reto de construir formas sostenibles de colaboración intercultural.

\section{Programas de "inclusión de individuos" indígenas y/o afrodescendientes como estudiantes en universidades u otros tipos de IES "convencionales"}

Existen numerosas experiencias que responden a este tipo de modalidad (programas de cupos, de becas y de apoyo académico y/o psicosocial), aunque aún resultan insuficientes en comparación con las necesidades y demandas. Varios Estados, universidades y otros tipos de IES públicas y privadas de América Latina han establecido programas de cupos y de becas, otro tanto han hecho varias fundaciones privadas. Hay opiniones encontradas respecto de este tipo de programas. Algunas enfatizan que no sólo crean oportunidades para individuos, sino que contribuyen a desarrollar capas de profesionales indígenas que sirven de manera directa a sus pueblos y comunidades, así como que muchos de estos profesionales juegan papeles fundamentales en el desarrollo y gestión de experiencias de los cuatro tipos restantes de esta clasificación. Algunas opiniones destacan que este tipo de programas favorecen la "fuga de cerebros" desde las comunidades apartadas hacia las grandes ciudades, así como su "occidentalización", la pérdida de sus valores étnicos, de sus lenguas y de la valoración por sus saberes. Otras señalan que con las becas no basta, porque en las IES "convencionales" estos estudiantes frecuentemente deben lidiar con problemas de racismo, además de que en el currículo no encuentran reflejadas las historias, idiomas y conocimientos de sus pueblos, a lo que se añade que se encuentran muy lejos de sus familias y comunidades. Algunos programas de inclusión de individuos combinan más de una estrategia de abordaje, por ejemplo combinan cupos con becas y/o con apoyo psicosocial, otros se complementan con seminarios transversales, o con cátedras libres orientadas a educar sobre el tema a la comunidad universitaria en su conjunto, o bien se articulan con proyectos de investigación y/o de extensión que se desarrollan conjuntamente con comunidades de pueblos indígenas y/o afrodescendientes.

Las características de las formas de colaboración intercultural en el marco de programas de "inclusión de individuos" son muy diversas. En algunos casos son complejas y elaboradas, en otros consisten solo en actividades que se presentan como "diálogos de saberes", las cuales frecuentemente se limitan a organizar mesas de trabajo en las que docentes o investigadores universitarios e intelectuales o referentes de alguna comunidad u organización indígena presentan sus puntos de vista sobre algún asunto en particular. Pero los formatos utilizados habitualmente solo permiten que apenas dialoguen amablemente. No permiten profundizar, ni menos aún plantearse actividades conjuntas en las que podrían construirse formas de colaboración intercultural sostenibles. Las iniciativas de este tipo no deben desvalorizarse, muchas veces son un valioso primer paso, pero es importante tener conciencia de sus limitaciones y buscar formas de avanzar hacia objetivos más ambiciosos.

\section{Programas de formación técnica o profesional (conducentes a títulos $u$ otras certificaciones) creados por universidades $u$ otras IES "convencionales"}

Hay diversas modalidades dentro de este grupo de experiencias. En algunas de ellas se registra escasa colaboración intercultural, mientras que en otras la participación de organizaciones y comunidades indígenas y/o afrodescendientes resulta significativa. En algunos de estos casos se constata además una participación importante de docentes provenientes de los mencionados pueblos y la inclusión de sus lenguas, conocimientos, formas de aprendizaje y modos de producción de conocimiento. Muchas de estas experiencias están orientadas a la formación de docentes para programas de educación intercultural bilingüe a diversos niveles del sistema educativo.

En algunas de estas experiencias, las personas indígenas o afrodescendientes no solo participan como estudiantes, sino que también se incorpora a sabios y ancianos de las comunidades para que contribuyan con sus conocimientos. En algunos casos su participación es solo ocasional, pero otras participan en calidad de docentes durante uno o más cursos. Junto con esto se presenta el problema de que por no contar con título universitario (y en ocasiones tampoco de otros niveles educativos) estos sabios no son formalmente reconocidos como docentes, ni remunerados como tales. Estas situaciones además de ser injustas constituyen nuevos mecanismos de subalternización de conocimientos y personas indígenas, y son fuentes de conflictos. Paradójicamente, estos referentes indígenas o afrodescendientes generalmente son los únicos pueden dar clases de idiomas indígenas, o sobre las respectivas historias y cosmovisiones, o al menos quienes mejor pueden hacerlo.

Muchos de programas de este tipo están dirigidos a docentes en servicio en comunidades indígenas o afrodescendientes. Aprovechando esta posibilidad, suelen combinar las actividades en las aulas universitarias con formas de enseñanza-aprendizaje en servicio en las escuelas de las comunidades en las cuales estos estudiantes universitarios son docentes. En otros casos, las clases a cargo de sabios indígenas se dan en los propios territorios, especialmente cuando el contenido de las mismas se relaciona 
con conocimientos etnobotánicos y otros en los cuales estar en territorio resulta especialmente provechoso. Estas estrategias de trabajo suelen abrir amplias posibilidades para desarrollar formas sostenibles de colaboración intercultural. No obstante, estas no están exentas de asimetrías de poder, dificultades y conflictos, todo lo cual es — de todos modos- parte de los caminos a transitar para poder construir modalidades sostenibles de colaboración intercultural, que sean equitativas y provechosas.

\section{Programas y proyectos de docencia, investigación ylo de} vinculación social, desarrollados por IES "convencionales" con participación de comunidades de pueblos indígenas y/o afrodescendientes

El conjunto de experiencias que forzadamente incluyo aquí es expresión de la diversidad de contextos, culturas profesionales y académicas, culturas institucionales, y normativas monoculturalistas, que ponen a prueba la creatividad de numerosos grupos de docentes, investigadores, autoridades universitarias y otros actores significativos. Por esto, bajo la complicada denominación de esta modalidad he agrupado muy diversas experiencias que, un tanto arbitrariamente, se podrían separar en tres subconjuntos, pero el problema es que algunas formarían parte de más de uno de estos. Las experiencias comprendidas en cualquiera de ellos pueden alcanzar logros más o menos significativos en el desarrollo de relaciones de colaboración intercultural, las cuales como en los casos antes comentados no están exentas de diferencias de intereses y visiones, asimetrías de poder, dificultades y eventualmente conflictos.

El primero de esos subconjuntos abarcaría las experiencias centradas principalmente en programas o proyectos de docencia, algunos de los cuales incluyen componentes de investigación y/o de vinculación con la comunidad. Se diferencian de los del tipo descrito en la sección anterior, en que se trata de una o más asignaturas o seminarios que no necesariamente otorgan créditos, puntos, u horas para graduarse. Dependiendo de los casos, este tipo de actividades se caracteriza por incluir la participación de docentes provenientes de los pueblos indígenas y la inclusión de lenguas, saberes y modos de producción de conocimiento y aprendizaje propios de estos pueblos.

El segundo subconjunto abarcaría experiencias en las cuales la colaboración intercultural se da principalmente a través de programas o proyectos de investigación, algunos de los cuales además incluyen actividades docentes, otras no. Según los casos, estas experiencias producen conocimientos sobre estas comunidades, sistematizan sus idiomas y conocimientos, o generan tecnologías, que comparten de manera proactiva con las comunidades, o bien hacen esto mismo pero mediante modalidades de coproducción.

El tercer subconjunto incluiría experiencias en las que la colaboración se da especialmente mediante proyectos denominados de "vinculación", "extensión", "servicio", u otras denominaciones, orientados a mejorar la calidad de vida de las comunidades. En muchos casos no se limitan a "aplicar" saberes académicos en las comunidades, sino que integran saberes de las comunidades. Este tipo de casos se caracterizaría porque aunque, incluye actividades docentes y de investigación, las principales son de servicio a y vinculación con las comunidades.

\section{Convenios de coejecución entre universidades u otros tipos de IES "convencionales" y organizaciones o comunidades indígenas y/o afrodescendientes}

Aunque se trata de una modalidad de trabajo potencialmente muy provechosa, las experiencias de este tipo son relativamente escasas. Algunas se conciben desde el principio para alcanzar metas relativamente limitadas y son de duración muy acotada, unos meses, un año, un par de años. Otras comienzan de esta forma pero su existencia si los convenios se renuevan su existencia se extiende. En tanto otras se conciben desde el principio para extenderse por varios años, algunas de ellas han dado lugar a la creación de programas especiales al interior de las universidades u otras IES. Todas ellas involucran desde el comienzo la necesidad de negociar intereses y objetivos. Todas las que han sido estudiadas desde los dos proyectos que sirven de referencia a este artículo han sido propuestas por organizaciones, comunidades o referentes indígenas o afrodescendientes a las universidades $u$ otros tipos de IES. Todas ellas han logrado satisfacer en mayor o menor medida los objetivos previstos. Esto de ningún modo supone que en el curso de las mismas no se hayan presentado diferencias y conflictos, sino que se ha logrado manejar estos sin comprometer los objetivos.

\section{Universidades y otros tipos de IES interculturales}

Las universidades y otros tipos de IES interculturales se caracterizan por integrar los saberes, modos de producción de conocimiento y modos de aprendizaje de varias tradiciones culturales. Más allá de este rasgo común, estos tipos de instituciones son muy diversos entre sí, como consecuencia de las diferencias existentes entre diversos pueblos indígenas o afrodescendientes, diversos Estados nacionales, diversos actores que han intervenido en su creación y otros factores. No existe un modelo de referencia, y dadas las diferencias apuntadas tampoco sería pertinente pretender establecerlo.

Como consecuencia del proceso de colonización y las continuidades del mismo observables en las repúblicas fundadas en el siglo XIX, estas instituciones han sido creadas especialmente para atender las demandas y propuestas de formación de pueblos indígenas y/o afrodescendientes. Lo cual de ningún modo puede considerarse "natural", ya que en sociedades pluriculturales la educación intercultural debería estar dirigida a todos los componentes de las mismas. 
El caso es que estas instituciones reciben principalmente estudiantes indígenas $\mathrm{y} / \mathrm{o}$ afrodescendientes y en mucho menor medida estudiantes que no se identifican como pertenecientes a estos pueblos. En vista del rezago de los Estados en satisfacer las demandas de "interculturizar toda la educación superior", dirigentes y organizaciones indígenas y afrodescendientes de varios países de la región se han dado a la tarea de crear instituciones propias. Por otra parte, también existen universidades y otros tipos de IES de carácter intercultural que han sido creadas por organismos estatales de distinto nivel, y otras que han sido creadas formando parte de una universidad o sistema universitario "convencional". También existe un caso particular, el de la Universidad Indígena Intercultural, que ha sido creada por un organismo multilateral particular, el Fondo para el Desarrollo de los Pueblos Indígenas de América Latina y el Caribe, el cual es cogobernado por un cuerpo de representantes de gobiernos y organizaciones indígenas. Las publicaciones de los dos proyectos que sirven de referencia a este artículo documentan la experiencia de varias universidades de cada uno de los tipos antes mencionados (Mato, 2008; 2009a; 2015; 2016). Limitaciones de extensión impiden exponer acá sobre las importantes diferencias existentes entre las universidades $u$ otras IES interculturales creadas por organizaciones $y / 0$ referentes indígenas $\mathrm{y} / \mathrm{o}$ afrodescendientes y las creadas por organismos estatales, sobre las cuales se ha expuesto en detalle en las publicaciones antes mencionadas. No obstante, de manera sintética cabe afirmar que en la mayoría de las universidades creadas por organizaciones $y / 0$ intelectuales de pueblos indígenas y/o afrodescendientes, el adjetivo "intercultural" alude a que incluyen conocimientos de diversos pueblos de estos tipos y también de la tradición occidental moderna. En esto se diferencian claramente del uso que los Estados hacen de este mismo adjetivo, ya que estos lo utilizan para referir a las relaciones entre dos "culturas" homogéneamente representadas, la de la "sociedad nacional" y "la indígena". Si bien suelen reconocer la existencia de diferencias al interior de esta última, en la práctica suelen presentarla como si fuera homogénea. En cualquier caso, a los efectos del foco de este texto, lo más importante es tener presente que dado su carácter intercultural, todas los tipos de universidades y otras IES incluidas en este último tipo de la tipología involucran el desarrollo de relaciones de colaboración intercultural entre actores diversos, en algunos más casos más diferenciados entre sí que en otros.

\section{Educación superior y pueblos indígenas y afrodescendientes: logros, problemas y desafíos}

Los ya mencionados dos proyectos de amplio alcance que sirven referencia a este artículo han permitido identificar tanto los principales logros de las experiencias estudiadas, como los problemas y desafíos que estas deben enfrentar. De manera sintética son los siguientes.

\section{Logros}

Los principales logros de los programas, universidades y otras IES estudiadas son: i) mejoran las posibilidades de que individuos indígenas y afrodescendientes accedan a oportunidades de educación superior y culminen exitosamente sus estudios, ii) ajustan su oferta educativa a necesidades, demandas y proyectos de las comunidades y la relacionan con oportunidades locales y regionales (subnacionales) de empleo, generación de iniciativas productivas y servicio a la comunidad, iii) desarrollan modalidades participativas de aprendizaje centradas en la investigación aplicada, iv) integran docencia/aprendizaje, investigación y servicio a las comunidades, v) integran diversos tipos de saberes y modos de producción de conocimiento, vi) promueven la valorización y, según los casos, incorporan lenguas y conocimientos propios de estos pueblos y comunidades, contribuyen proactivamente a su fortalecimiento y realizan investigación sobre ellos, vii) desarrollan docencia e investigación orientados por criterios de valoración de la diversidad cultural, interculturalidad, equidad, inclusión, gobernabilidad democrática, desarrollo humano y sostenible, viii) forman egresados que contribuyen al desarrollo sostenible local y regional y al mejoramiento de la calidad de vida de sus comunidades.

\section{Problemas y desafíos}

Los problemas y desafíos más frecuentemente enfrentados por estas universidades, IES y programas son: i) insuficiencia y/o precariedad presupuestaria, ii) actitudes racistas por parte de funcionarios públicos y diversos sectores de población que afectan el desarrollo de sus actividades, iii) dificultades derivadas de la rigidez de los criterios de evaluación aplicados por las agencias especializadas de los Estados encargadas de otorgar reconocimiento y/o acreditación, iv) obstáculos institucionales derivados de la rigidez de procedimientos administrativos, los cuales afectan la ejecución de sus planes y actividades, v) obstáculos institucionales derivados de la rigidez de criterios académicos aplicados por las IES dentro de las cuales funcionan algunos de los programas estudiados, y/o por agencias gubernamentales que otorgan fondos para investigación y actividades de formación, vi) dificultades para conseguir docentes y otro personal con adecuada sensibilidad y recursos personales y técnicos para el trabajo intercultural, vii) dificultades económicas de los estudiantes para poder dedicarse más y mejor a su formación, viii) insuficiencia de becas. 
es necesario diferenciar entre la expresión genérica "diálogo de saberes",a veces

aplicada de manera bastante ingenua y

como si con solo invocarla se abriera un espacio de idílica armonía

Consideraciones finales: retomar los problemas y desafíos en la construcción de modalidades duraderas y provechosas de colaboración intercultural

Los avances desde la invocación de la expresión "diálogo de saberes", que suele servir de inspiración o primer paso a muchas iniciativas en el campo que nos ocupa, hacia la construcción de modalidades duraderas y provechosas de colaboración intercultural en docencia, investigación y/o extensión, suelen darse a través de una compleja trama de dificultades, negociaciones y conflictos, no solo entre actores claramente diferenciados (por ejemplo entre universidades y organizaciones, comunidades o referentes de pueblos indígenas), sino frecuentemente al interior de las propias universidades, entre diversos sectores de las mismas, así como con las agencias gubernamentales que acreditan sus actividades, formulan políticas y otorgan fondos para su funcionamiento.

En ocasiones el llamado "diálogo de saberes" no pasa de la mutua escucha de las exposiciones de conocimientos entre académicos y personas de conocimiento, ancianos o dirigentes de las comunidades. En otros, en cambio, la organización de eventos de este tipo responde a una estrategia de construcción más ambiciosa, en la cual aún no se ha logrado avanzar mucho, debido a que esto, como sabemos, toma tiempo y esfuerzos. Escucharnos mutua, concienzuda, sensible y respetuosamente es sin duda muy importante y una condición imprescindible para poder "avanzar" hacia modalidades sostenibles de colaboración intercultural que sean mutuamente respetuosas y equitativas, y no solo eso, sino que contribuyan a construir sociedades más democráticas. Estas formas de colaboración son necesarias para el desarrollo de experiencias duraderas y provechosas de producción y aplicación de conocimientos, para el aprendizaje de conocimientos, el desarrollo de destrezas y competencias, y para la puesta en práctica de acciones en beneficio de comunidades de pueblos indígenas y de la sociedad en general, las cuales - no puede omitirse - también benefician a las universidades.

Las modalidades de colaboración intercultural a las que hago referencia no son una quimera, existen. Muchas de ellas son características de algunas de las experiencias en este campo actualmente existentes a las que he hecho referencia en la sección 3 de este mismo artículo. Los retos ahora son de dos tipos. Por un lado es necesario diferenciar entre la expresión genérica "diálogo de saberes", a veces aplicada de manera bastante ingenua y como si con solo invocarla se abriera un espacio de idílica armonía, y la construcción de modalidades duraderas y provechosas de colaboración intercultural, lo cual entre otras cosas demanda reconocer la existencia de diferencias, inequidades y conflictos. Por otro lado, se trata de lograr la profundización, valoración, apoyo y reconocimiento institucional tanto de las universidades (y otros tipos de IES) indígenas, afrodescendientes, interculturales y comunitarias actualmente existentes, como de los tipos de 
acciones que suelen ser impulsadas en el marco de los programas especiales de formación en universidades convencionales a que hemos hecho referencia en la tipología antes presentada, como también en los programas de inclusión de individuos (que algunos actores en este campo llaman "de acción afirmativa", traduciendo la expresión en inglés affirmative action), así como en los proyectos y programas impulsados por cátedras, departamentos, centros e institutos de investigación y en muchos casos incluso por colegas individuales o grupos de colegas, en los cuales —según los casos- trabajan en colaboración con personas, comunidades $y / u$ organizaciones indígenas en labores de extensión, docencia y/o investigación.

Estos variados tipos de experiencias de colaboración intercultural pueden servir de referencia para desarrollar estrategias de trabajo adecuadas a los diversos contextos sociales e institucionales. No resulta recomendable tratar de seguir el formato de supuestas "buenas" o "mejores" prácticas, sino aprender de los logros y dificultades de experiencias propias y ajenas para desarrollar nuevas experiencias, cónsonas con las propuestas de los actores participantes y pertinentes respecto de los contextos específicos en que se procura crearlas. Con estos objetivos en vista, me parece conveniente agregar algunas palabras sobre cuatro de los problemas y desafíos mencionados en la sección anterior:

i) Insuficiencia y/o precariedad presupuestaria: los problemas derivados de insuficiencias presupuestarias puede que resulten obvios. Pero quienes no participan directamente de este tipo de experiencias, tal vez no logren dimensionar lo inmensamente dañina que puede resultar la precariedad presupuestaria no solo para cualquier proyecto, sino especialmente para aquellos que se desarrollan en colaboración con comunidades sociales que ya conocen una larga historia de incumplimientos por parte de las instituciones del Estado, universidades incluidas. Que no solo el inicio, sino también la continuidad de las actividades desarrolladas con estas comunidades (sean de formación, extensión, aprendizaje-servicio, o de investigación) dependa de convocatorias anuales de fondos que pueden ganarse o no, dependiendo de evaluaciones que quienes estamos en tema en no pocas ocasiones consideramos cuestionables, que además esos fondos usualmente sean muy insuficientes y que para peor en ocasiones se reciban con atraso, conspira contra cualquier posibilidad de hacer un trabajo serio y de ser dignos de la confianza y el respeto de las comunidades con las cuales trabajamos. Para lograr avanzar en la construcción de modalidades sostenibles de colaboración intercultural con comunidades de pueblos indígenas y/o afrodescendientes es necesario contar con presupuestos suficientes y regulares. Es necesario lograr que los tomadores de decisiones comprendan y valoren la importancia de este tipo de problemas. Este tipo de problemas debe encararse otorgándole la importancia crucial que tiene y disponiéndonos a llevar los planteos del caso a todas las instancias necesarias. Esto demanda abordarlo con la densidad ética, política y académica que amerita. Es necesario que algunos de nuestros proyectos de investigación y los artículos resultantes se dediquen a hacer visible este tipo de problemas y sus consecuencias. Es necesario formar opinión al respecto, para esto puede resultar provechoso escribir sobre el tema en medios de comunicación universitarios. También puede ser útil enviar comunicaciones directas a los órganos de gobierno universitario y a las agencias gubernamentales que otorgan fondos para Educación Superior y Ciencia y Técnica. El presupuestario no es un problema menor. Debe ser un punto central en las deliberaciones sobre el tema y pasar a formar parte de las agendas compartidas entre quienes trabajamos en estos tipos de experiencias. Si no se logra avanzar en esta materia de poco sirven las reflexiones epistemológicas, éticas y políticas que podamos hacer en las aulas o en nuestras publicaciones.

\section{ii) Dificultades derivadas de la rigidez de los criterios} de evaluación aplicados por las agencias especializadas de los Estados encargadas de otorgar reconocimiento y/o acreditación: este es un problema muy importante respecto del cual han venido insistiendo diversos actores significativos. Las universidades indígenas e interculturales, como los programas especiales con/para pueblos indígenas y/o afrodescendientes no pueden ser evaluadas con los mismos criterios que las universidades "convencionales", sus facultades y carreras. No porque sean de "menor calidad", sino porque responden a otros objetivos, es decir, porque son cualitativamente diferentes de las "convencionales". Del mismo modo que las carreras de ingeniería no pueden ser evaluadas con los mismos criterios que las de medicina, o viceversa, ni las derecho con los mismos criterios que las de agronomía, o viceversa. Los problemas radican tanto en los protocolos de evaluación establecidos por las instituciones, como en los frecuentemente escasos o nulos conocimientos de los "pares evaluadores" respecto de asuntos relativos a diferencias y relaciones interculturales, colaboración intercultural y otros conexos, y las consecuentes limitaciones al respecto de su sensibilidad, "visión" y criterios. Un ejemplo significativo puede ser el de las limitaciones impuestas por los protocolos de evaluación de las instituciones que impiden valorar que si bien 
las universidades indígenas no suelen contar con bibliotecas poseedoras de grandes colecciones de publicaciones en varios idiomas, en cambio cuentan con los conocimientos de sabios indígenas y otros de tradición oral que circulan en sus respectivos territorios. Estos problemas en general están asociados a las ideas hegemónicas de "calidad académica" que sobrevaloran las publicaciones en revistas académicas, especialmente si son internacionales y de "alto impacto". En cambio, no valoran la importancia y beneficios para las universidades de las experiencias de vinculación social, como espacios de formación de los estudiantes, y de identificación de temas de investigación y desarrollo de actividades de investigación de docentes, investigadores y los propios estudiantes.

\section{iii) Obstáculos institucionales derivados de la rigidez de} criterios académicos aplicados por las universidades dentro de las cuales funcionan algunos de los programas estudiados, y/o por agencias gubernamentales que otorgan fondos para investigación o formación: este tipo de obstáculos resulta bastante semejante al antes mencionado, pero lo menciono separadamente porque dificulta la posibilidad de sumar las contribuciones de sabias/os indígenas y/o afrodescendientes que no poseen títulos universitarios, tanto en actividades docentes como de investigación. Este problema no es menor. Refuerza asimetrías de poder, deslegitima los conocimientos de los pueblos indígenas y/o afrodescendientes, al hacerlo respecto de estos especialistas no universitarios, quienes en muchos casos poseen conocimientos tanto o más valiosos que sus contrapartes universitarias. Muchos de quienes trabajamos con estos colegas indígenas $\mathrm{y} / \mathrm{o}$ afrodescendientes hemos tenido que confrontar respecto de este tema con las burocracias universitarias, y/o de agencias gubernamentales. Este, como los dos tipos de dificultades anteriores, demanda que nos comprometamos proactivamente en hacer investigación que muestre los problemas generados, así como en argumentar sobre el tema en todos los foros posibles. La construcción de modalidades sostenibles de colaboración intercultural exige este tipo de labor, no puede ignorarse. La apropiada valoración de este tipo de modalidades que van - digamos - a contrapelo de la institucionalidad establecida y naturalizada como "sentido común" demanda que se realice investigación sobre estos temas. No es algo opcional.

\section{Referencias bibliográficas}

Almeida, M.; Marmet, M.; Strada, V.; Zuttión, B. y Cabrera, Z. (2011). Producción de saberes y extensión. Ponencia presentada en el XI Congreso Iberoamericano de Extensión Universitaria "Integración extensión, docencia e investigación para la inclusión y cohesión social", Santa Fe, 22 al 25 de noviembre.

Banco Mundial (2015). Latinoamérica indígena en el siglo XXI: primera década. Washington DC: Banco Mundial.

CIDH-OEA-Comisión Interamericana de Derechos Humanos (2011). La situación de las personas afrodescendientes en las Américas. (OEA/Ser. LN/II. Doc. 62, 5 diciembre 2011. Original: Español): Washington DC: OEA.

IIPE-UNESCO-Instituto Internacional de Planeamiento de la Educación (2012) La situación educativa de la población indígena y afrodescendiente en América Latina. Buenos Aires: IIPE-UNESCO.

FAUBA (Facultad de Agronomía, Universidad de Buenos Aires) (2015). Libro de resúmenes de la VI Jornada de Difusión de Actividades de Extensión en la FAUBA "La extensión y la investigación, procesos que se retro-alimentan". Buenos Aires, 6 de noviembre. Disponible en: https://www.agro.uba.ar/sites/default/files/libro_ de_resumens_jornada_2015.pdf

Macas, L. (2001). Diálogo de culturas. Hacia el reconocimiento del otro. En: Yachaykuna (Instituto Científico de Culturas Indígenas), 2, 44-55.

Mato, D. (2013a). Aprendizajes de equipos universitarios en experiencias de colaboración con comunidades y organizaciones sociales, realizadas con apoyo del Programa de Voluntariado Universitario de Argentina, en 2008. Apuntes. Revista de Ciencias Sociales (Universidad del Pacífico, Perú), XL(72), 33-56.

_ (2013b). Contribución de experiencias de vinculación social de las universidades al mejoramiento de la calidad académica y factores que limitan su desarrollo y valoración institucional. Revista Avaliação: Revista da Avaliação da Educação Superior (Universidade de Sorocaba, Brasil), 18(1), 151-180.

(2015). Vinculación social universitaria en Argentina. Diversidad de orientaciones de trabajo, logros y dificultades de las experiencias apoyadas por el Programa Nacional de Voluntariado Universitario. CPU-e, Revista de Investigación Educativa (Universidad Veracruzana, México), 20, 132-149.

Mato, D. (Coord.) (2008). Diversidad cultural e interculturalidad en Educación Superior. Experiencias en América Latina. Caracas: UNESCO-IESALC.

- (2009a). Instituciones Interculturales de Educación Superior en América Latina. Caracas: UNESCO-IESALC.

— (2009b). Educación Superior, Colaboración Intercultural y Desarrollo Sostenible/Buen Vivir. Experiencias en América Latina. Caracas: UNESCO-IESALC. - (2012). Educación Superior y Pueblos Indígenas y Afrodescendientes en América Latina. Normas, Políticas y Prácticas Caracas: UNESCO-IESALC. - (2016). Educación Superior y Pueblos Indígenas en América Latina. Experiencias, interpelaciones y desafíos. Sáenz Peña: EDUNTREF y México DF: Universidad Nacional Autónoma de México-UNAM.

(2017). Educación Superior y Pueblos Indígenas y Afrodescendientes en América Latina. Políticas y prácticas de inclusión, democratización, e interculturalización. Sáenz Peña: EDUNTREF.

Universidad Intercultural Amawtay Wasi (2004). Sumak Yachaypi, Alli Kawsay pipash Yachakuna / Aprender en la Sabiduría y el Buen Vivir / Learning Wisdom and the Good Way to Live. Quito: Universidad Intercultural Amawtay Wasi. 